Benchmarks

\title{
A novel mixing device for the reproducible generation of nonviral gene therapy formulations
}

Lee A. Davies ${ }^{1,4}$, Graciela A. Nunez-Alonso ${ }^{1,4}$, Henry L. Hebel ${ }^{2}$, Ron K. Scheule ${ }^{3}$, Seng H. Cheng ${ }^{3}$, Stephen C. Hyde ${ }^{1,4}$, and Deborah R. Gill ${ }^{1,4}$

${ }^{1}$ Gene Medicine Group, Nuffield Department of Clinical Laboratory Sciences, University of Oxford, John Radcliffe Hospital, Oxford, UK, ${ }^{2} V G X I$, Houston, TX, USA, ${ }^{3}$ Genzyme Corporation, Framingham, MA, USA, and ${ }^{4}$ The UK Cystic Fibrosis Consortium

BioTechniques 49:666-668 (September 2010) doi 10.2144/000113498 Keywords: Gene therapy, nonviral formulations, lipoplexes, polyplexes

Nonviral gene therapy utilizing plasmid DNA (pDNA) complexed with cationic lipids (lipoplexes) or cationic polymers (polyplexes) has demonstrated considerable potential for the treatment of a variety of diseases. However, progress toward clinical application is often delayed by the lack of reliable and scalable mixing of components sufficient to guarantee consistent performance in vivo. Attempts to improve and standardize mixing have been limited by the sensitivity of pDNA to shear-related degradation. Here we describe a simple pneumatic mixing device that enables the rapid and reproducible production of large volumes of nonviral gene therapy formulations and demonstrate its suitability for use with shearsensitive pDNA.

Due to the susceptibility of plasmid DNA (pDNA) to shear-related degradation (1), nonviral gene therapy formulations are typically prepared in the laboratory in relatively small volumes $(<1 \mathrm{~mL})$ by simply adding pDNA to a solution of lipid or polymer with subsequent mixing by repeated inversion or pipetting. Under such conditions, mixing parameters are virtually impossible to standardize leading to variability in the physical characteristics of complexes prepared in different laboratories, even when using identical starting material (2). Such variability is further exacerbated by attempts to scale-up mixing volumes for pre-clinical and clinical studies where differences in physical characteristics may crucially impact key in vivo outcomes including gene expression, toxicity, and biodistribution (3). While some attempts have been made to standardize mixing within the laboratory (2), many of the handmixing devices remain prone to operator variability and are unlikely to prove acceptable in a clinical setting.

To address these issues, we have developed a simple automated mixing device, the LMD 2 (Figure 1A), that utilizes compressed air to depress the plunger of a disposable dualbarrel polypropylene syringe (Plas-Pak Industries, Norwich, CT, USA) containing pDNA and lipid/polymer in separate compartments (Figure 1B). Activation of the device initiates mixing of the components by simultaneous extrusion of the two reagents through an 8-element HDPE divide/merge static mixer device (Cat. no. 003M08B005; Plas-Pak) affixed to the outlet of the syringe. To generate the relatively high mixing speeds required for complex formation, the device utilizes a cylindrical pneumatic actuator (SMC Pneumatics, Milton Keynes, UK) connected via an adjustable supply regulator to a 50-psi compressed air supply. The supply regulator modulates the speed of the pneumatic plunger, making it possible to accurately control the mixing rate of reagents and enabling critical mixing
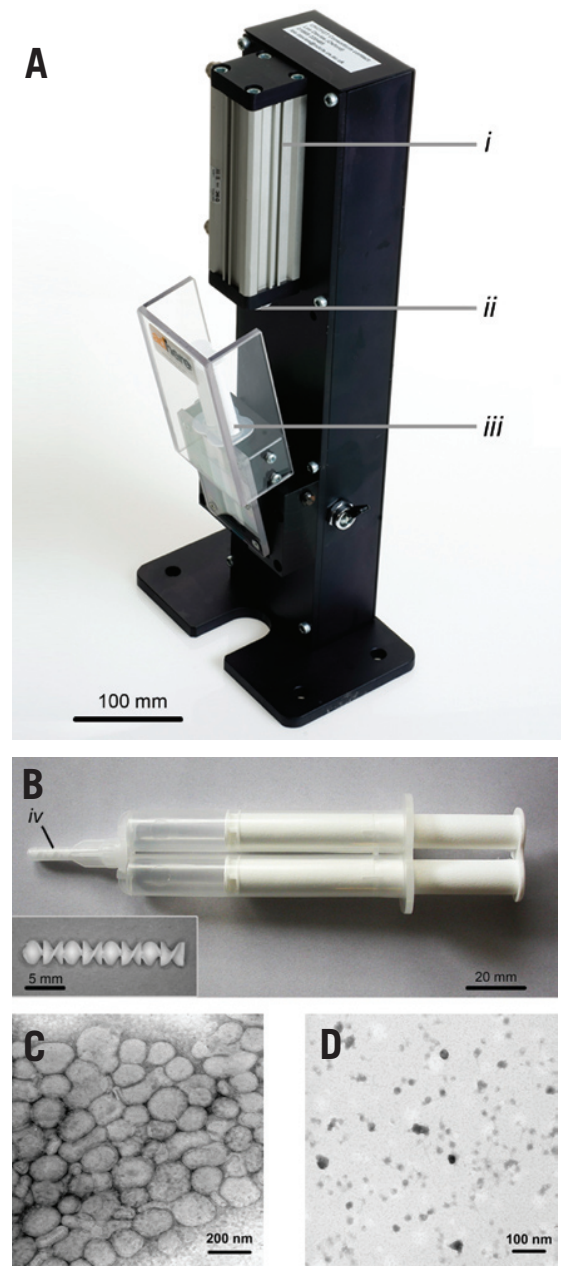

Figure 1. The LMD 2 pneumatic mixer. (A) The LMD 2 mixing device incorporating a pneumatic actuator (i), plunger (ii), and dual-lumen mixing syringe (iii). (B) Dual lumen mixing syringe incorporating 8-element static mixer (iv). Insert contains magnified view of the exposed static mixer elements. (C) Electron micrographs of pDNA/GL67A complexes and (D) pDNA/PEI complexes.

parameters including the Reynolds number and shear rate to be defined and optimized for individual formulations. While the described LMD 2 model can prepare a single dose of $\leq 14 \mathrm{~mL}$ formulation, simple modifications to the LMD 2 design permit the use of smaller or larger dual-lumen syringes suitable for the production of $1-100 \mathrm{~mL}$ per actuation. The degree of component mixing can also be varied by inclusion of additional static mixer elements and as such, the device is useful for a wide variety of applications.

To investigate the viability of this mixing device, the LMD 2 equipped with a $2 \times 7 \mathrm{~mL}$ dual-barrel syringe (Cat. no. 014B35X001; Plas-Pak) was used to complex the $5.6-\mathrm{kb}$ luciferase expression plasmid pCIKLux (4) with two disparate nonviral gene transfer agents that have shown promise for lung gene therapy. 
Table 1. Physical characteristics of nonviral gene therapy formulations.

\begin{tabular}{|c|c|c|c|c|c|c|c|}
\hline & & \multicolumn{6}{|c|}{ Mixing rate $(\mathrm{mL} / \mathrm{s})$} \\
\hline \multirow{3}{*}{ 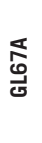 } & Reynolds number ${ }^{a}$ & $\mathrm{~N} / \mathrm{A}$ & 544 & 1090 & 2720 & 5440 & 10,900 \\
\hline & Hydrodynamic diameter (nm) & $265 \pm 18$ & $287 \pm 13$ & $278 \pm 13$ & $285 \pm 19$ & $306 \pm 16^{* *}$ & $315 \pm 22^{* *}$ \\
\hline & Zeta potential $(\mathrm{mV})$ & $+3.8 \pm 0.2$ & $+3.5 \pm 0.3$ & $+3.6 \pm 0.4$ & $+3.4 \pm 0.3$ & $+4.1 \pm 0.5$ & $+4.1 \pm 0.4$ \\
\hline च & Zeta potential $(\mathrm{mV})$ & $+47.3 \pm 0.7$ & $+47.1 \pm 0.9$ & $+48.3 \pm 0.4$ & $+48.8 \pm 0.3$ & $+49.9 \pm 0.4$ & $+48.4 \pm 0.8$ \\
\hline
\end{tabular}

Hydrodynamic diameter and zeta potential of pCIKLux/GL67A (molar ratio 0.6 mM: $0.8 \mathrm{mM}$ ) and pCIKLux/PEI (N:P ratio of 10:1) formulations prepared using the LMD 2 over a range of mixing rates. Data represents mean \pm SEM for $n=3$ replicates for each mixing condition measured using a Zetasizer Nano (Malvern Instru-

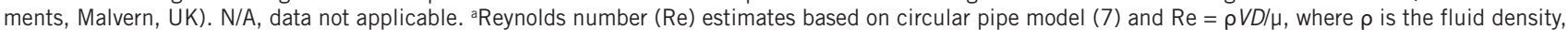
$V$ is fluid velocity, $D$ is the pipe diameter and $\mu$ is the fluid dynamic viscosity. Re values $<2000$ are indicative of laminar flow characteristics and values $>2000$ indicative of turbulent flow (7). ${ }^{* *}$ indicates statistical difference compared with handmixed formulations $(P<0.05$ ANOVA with Dunnett's post-hoc analysis).

A

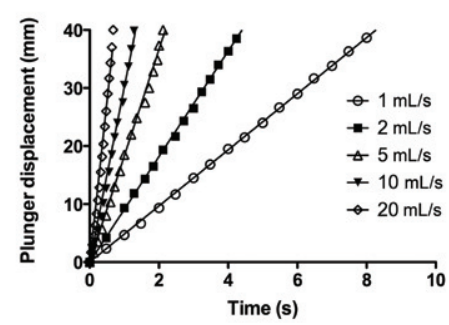

B

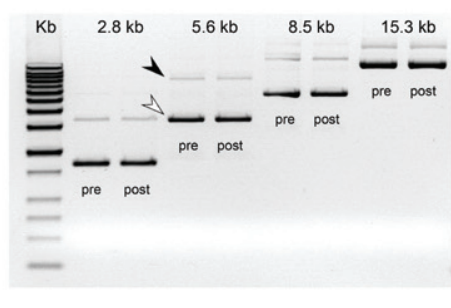

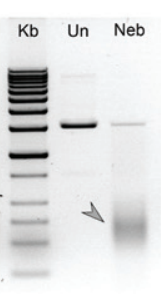

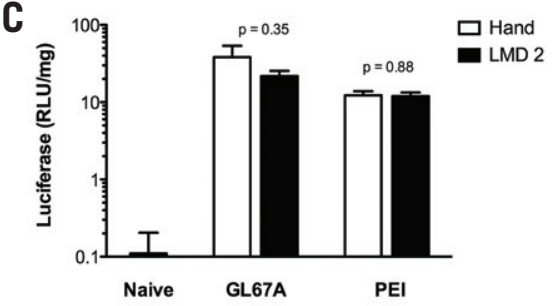

Figure 2. Preparation of gene therapy formulations using the LMD 2. (A) Constant and reproducible mixing rate using the LMD 2. Vertical displacement of the LMD 2 pneumatic plunger was measured using high-speed video analysis during mixing of pCIKLux/GL67A (6 mM: $8 \mathrm{mM}$ ) formulations over a range of volumetric mixing rates. Data represents mean \pm SEM of 3 replicates at each mixing rate. (B) Absence of shear degradation using the LMD 2. Conformational agarose gel analysis of pDNA following passage of plasmids from $2.1 \mathrm{~kb}$ to $15.3 \mathrm{~kb}$ through the LMD 2 at a volumetric mixing rate of $20 \mathrm{~mL} / \mathrm{s}$. Samples of pDNA before (pre) and after (post) mixing were analyzed. Open circular (black arrow) and closed covalent circular (white arrow) plasmid forms are indicated for the 5.6-kb plasmid only. For comparison, samples of the 5.6-kb plasmid were analyzed before (Un) and following shear degradation in a pneumatic nebulizer (Neb). Grey arrow indicates sheared DNA fragments of $\sim 0.5-0.75 \mathrm{~kb}$ in size. Kb indicates linear DNA size markers (Stratagene, Cambridge, UK). (C) In vivo performance of LMD 2 formulations. Luciferase expression in the lungs of BALB/c mice $24 \mathrm{~h}$ following aerosol delivery of $10 \mathrm{~mL} \mathrm{pCIKLux/GL67A} \mathrm{(6} \mathrm{mM:} 8 \mathrm{mM})$ or pCIKLux/PEI (N:P ratio of 10:1) prepared by hand or using the LMD 2. Data represents mean \pm SEM for $n=6$ mice in each treatment group. Statistical analysis performed using Student's $t$-test with $p$ values indicated.

GL67A (GL67:DOPE:DMPE-PEG $\left.{ }_{5000}\right)$ (5) is a cationic lipid that forms complex multi-lamellar lipoplexes with pDNA (Figure 1C) while 25-kDa polyethyleneimine (PEI) (6) is a synthetic polymer that forms small compact polyplexes with pDNA (Figure 1D). A total of $10 \mathrm{~mL}$ each formulation was prepared by mixing equal volumes of reagents using the LMD 2 operating at volumetric flow rates in the range of $1-20 \mathrm{~mL} / \mathrm{s}$. Importantly, highspeed video analysis of the mixing process demonstrated that at each flow rate tested, the plunger velocity-and hence the mixing rate within the device-was constant and highly reproducible (Figure 2A), even when relatively viscous formulations $(>20 \mathrm{cP})$ were utilized.

To investigate the effects of mixing rate on the physical characteristics of gene transfer formulations, hydrodynamic diameter and zeta potential were measured for complexes prepared over a range of mixing rates and compared with complexes prepared by standard mixing of small aliquots $(<1 \mathrm{~mL})$ by hand (Table 1$)$. Initial validation studies utilizing an alternative pDNA/PEI formulation, demonstrated the reproducibility of the LMD 2 at all mixing rates tested with both size and zeta potential measurements associated with relative variability $(\mathrm{CV})$ values in the range $5-12 \%$. Typical data $(n=33$ replicates) were observed using a mixing rate of $1 \mathrm{~mL} / \mathrm{s}$ where size $=+52.8 \pm 1.2 \mathrm{~nm}(\mathrm{CV}$ $=11.2 \%)$ and zeta potential $=52.4 \pm 0.9$ $\mathrm{mV}(\mathrm{CV}=9.5 \%)$. Importantly, production of large volumes $(10 \mathrm{~mL})$ of GL67A and PEI complexes at lower fluid mixing rates recapitulated exactly the physical characteristics observed with hand mixing. However, at higher mixing rates, significant increases in hydrodynamic diameter were observed for both reagents; this is possibly due to aggregation of complexes under increasingly turbulent mixing conditions, as indicated by calculated Reynolds numbers >>2000 (7) (Table 1). These results demonstrate the importance of a fully adjustable mixing device to enable selection of optimized mixing conditions for a given gene transfer agent.

While volumetric flow rate is a key variable in controlling the mixing of components within the LMD 2 it is also a critical factor in determining the shear to which pDNA is exposed. Estimates of generated shear rates based on the geometry of the LMD 2 static mixer element (calculated using $\gamma=4 V / \pi r^{3}(7)$, where $\gamma$ is shear rate, $V$ is volume velocity, and $r$ is the smallest inner radius within the device) range from $3,000 \mathrm{~s}^{-1}$ at $1 \mathrm{~mL} / \mathrm{s}$ to $30,000 \mathrm{~s}^{-1}$ at $20 \mathrm{~mL} / \mathrm{s}$. These values are lower than many common bioprocessing systems (8) and would suggest that the LMD 2 is a relatively low-shear device suitable for the mixing of delicate biomolecules including pDNA. This was confirmed by analysis of the physical integrity of a range of differently sized pDNA following mixing at 20 $\mathrm{mL} / \mathrm{s}$. No evidence of shear-related degradation was observed for any plasmid (Figure $2 \mathrm{~B}$ ), with complete retention of the biologically relevant (9) covalently closed circular 
Easy Way

for

\section{Your Life Science Research!}

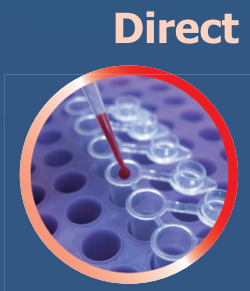

PCR Buffer

- No DNA Purification required - Save Time and Cost

- Less Risk of DNA Contamination - No Loss of Samples

- Compatibility with any other Taq - Direct PCR from Blood, Mouse tail

\section{Transfection Reagent}

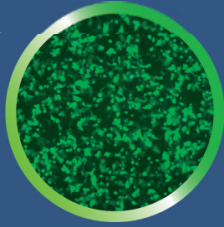

- Low Cellular Toxicity

- High Transfection Efficiency

- Efficient siRNA \& DNA delivery

- Transfection of a variety of cells

- Transfection into Stem Cells

- Ready-to-use reagent

\section{Cytokine ELISA Kit}

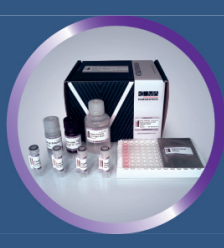

-EGF $\quad$ MCP-1 $\quad$-SRANKL

-FGF $\quad$ M-CSF $\quad$ TGF

-GM-CSF •MIP -TNF

-IFN NGF-beta -TRAIL

-IGF •PDGF-BB •VEGF

-Interleukin •RANTES

- Leptin ·SCF

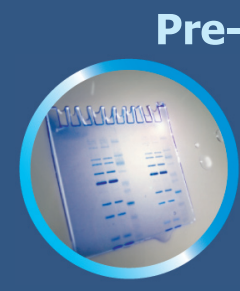

\section{Pre-Cast Gels}

- Long Life EzWay ${ }^{\top M} \mathrm{Ge}$

- Tris-Glycine SDS Gel

- Tris-Glycine non-SDS Gel

- Tricine Gel

- IEF Gel

-Zymogram Gel

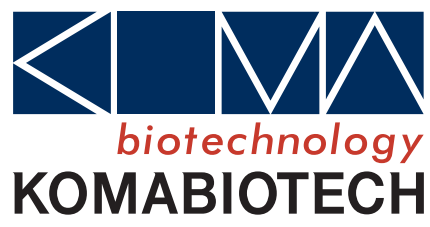

for more information www.komabiotech.com and open-circular plasmid forms (Figure $2 \mathrm{~B})$, even when relatively large plasmids were examined.

To confirm that formulations prepared using the LMD 2 were functional in vivo, $\mathrm{BALB} / \mathrm{c}$ mice were exposed to aerosols of pCIKLux/GL67A or pCIKLux/PEI generated using the LMD 2 at a mixing rate of $5 \mathrm{~mL} / \mathrm{s}$, or by combining multiple small aliquots $(<1 \mathrm{~mL})$ mixed by hand. Equivalent gene expression was observed for formulations prepared by hand or using the LMD 2 (Figure 2C) demonstrating that gene therapy formulations prepared using the LMD 2 retained full biological function in vivo.

In summary, we have developed a simple, practical mixing device that offers significant improvements over current production methodologies. The LMD 2 is quick, easy to use with a wide variety of reagents, and directly scalable for the production of formulations in the laboratory or in the clinic. All components in direct contact with experimental formulations are disposable, suitable for sterilization via gamma irradiation, and readily available. The LMD 2 device itself may be sanitized directly by application of pharmacyapproved detergent and/or alcohol-based cleaning products. Most importantly, the LMD 2 enables the standardized and highly reproducible production of nonviral gene therapy formulations. The device was recently approved for clinical production of gene therapy formulations and is currently being utilized in a Phase I clinical study in cystic fibrosis patients (http://clinicaltrials. gov/ct2/show/NCT00789867). Although the device is not currently commercially available, researchers wishing to obtain an LMD 2 should contact the authors directly.

\section{Acknowledgements}

The authors would like to thank Steve Porter (Plas-Pak) for his help and advice in the selection of appropriate mixing syringes and Paul Whitehead (Intertronics, Kidlington, UK) for his assistance and construction of the LMD 2 device. This work was funded by a grant from the UK Cystic Fibrosis trust (Bromley, UK) to the UK Cystic Fibrosis Gene Therapy Consortium (http:// cfgenetherapy.org.uk).

\section{Competing interests}

The authors declare no competing interests.

\section{References}

1.Levy, M.S., I.J. Collins, S.S. Yim, J.M. Ward, H.N. Titchener-Hooker, P.A. Shamlou, and P. Dunnill. 1999. Effect of shear on plasmid DNA in solution. Bioprocess Eng. 20:7-13.

2.Hirota, S., C.T. de Ilarduya, L.G. Barron, and F.C. Szoka, Jr. 1999. Simple mixing device to reproducibly prepare cationic lipid-DNA complexes (lipoplexes). Biotechniques 27:286-290.

3. Hattori, Y. and M. Hashida. 2005. Evaluation of Size and Zeta Potential of DNA/ Carrier Complexes, p. 293-299. Non-viral Gene Therapy. Springer, Tokyo.

4.Gill, D.R., S.E. Smyth, C.A. Goddard, I.A. Pringle, C.F. Higgins, W.H. Colledge, and S.C. Hyde. 2001. Increased persistence of lung gene expression using plasmids containing the ubiquitin $\mathrm{C}$ or elongation factor 1alpha promoter. Gene Ther. 8:1539-1546.

5. Eastman, S.J., M.J. Lukason, J.D. Tousignant, H. Murray, M.D. Lane, G.J. St, G.Y. Akita, M. Cherry, et al. 1997. A concentrated and stable aerosol formulation of cationic lipid:DNA complexes giving high-level gene expression in mouse lung. Hum. Gene Ther. 8:765-773.

6. Densmore, C.L., F.M. Orson, B. Xu, B.M. Kinsey, J.C. Waldrep, P. Hua, B. Bhogal, and V. Knight. 2000. Aerosol Delivery of Robust Polyethyleneimine-DNA Complexes for Gene Therapy and Genetic Immunization. Mol. Ther. 1:180-188.

7. Perry, R.H., D.W. Green, and J.O. Maloney. 1986. Perry's Chemical Handbook. McGrawHill, New York.

8. Lengsfeld, C.S. and T.J. Anchordoquy. 2002. Shear-induced degradation of plasmid DNA. J. Pharm. Sci. 91:1581-1589.

9. Cupillard, L., V. Juillard, S. Latour, G. Colombet, N. Cachet, S. Richard, S. Blanchard, and L. Fischer. 2005. Impact of plasmid supercoiling on the efficacy of a rabies DNA vaccine to protect cats. Vaccine 23:19101916.

Received 3 June 2010; accepted 23 July 2010.

Address all correspondence to Deborah R. Gill, Gene Medicine Group, Nuffield Department of Clinical Laboratory Sciences, University of Oxford, John Radcliffe Hospital, Oxford, UK. E-mail: deborah.gill@ndcls.ox.ac.uk 